\title{
Using market schedules to improve secondary control design
}

\author{
A. Virag* Student Member, IEEE, A. Jokić $\dagger$, P.P.J. van den Bosch*, P.M.J. Van den Hof Fellow, IEEE* \\ *Department of Electrical Engineering, Eindhoven University of Technology, Den Dolech 2, 5612 AZ Eindhoven, \\ The Netherlands, contact e-mail a.virag@tue.nl \\ †University of Zagreb, I. Lučića 5, 10000 Zagreb, Croatia
}

\begin{abstract}
Trends in the power system's development, such as increased demand response or penetration of renewable energy, indicate that in the future, power system will experience significant daily changes in their physical properties. Time frames of energy economics and physical processes start to overlap. These changes require proper secondary controller tuning, for which high-quality up-to-date models are needed. The main contribution of this paper, we utilize the information on the energy market outcome, which is known in advance, to identify the dynamics of a control area relevant for the secondary control (SC) design/tuning. By rethinking the interconnected power systems in the system identification framework, and exploiting the developed theory, a consistent model of a control area is obtained within a few hours. This is sufficient to follow the daily changes in the system dynamics. It is expected that a controller based on a better model adapts to the daily changes in power system dynamics, and moreover, is capable of improving the system performance. Several illustrative examples confirm that the use of market based signals in the identification process is an important step that helps getting accurate models.
\end{abstract}

\section{INTRODUCTION}

A power system is a constantly evolving, time-varying system. Society is witnessing the constant increase in the system's complexity and uncertainty. Changes have been introduced by (i) the increased demand response, such as electric vehicles or smart houses, and by (ii) a different generation mix with higher ratio of renewable energy, e.g., wind farms or solar panels. These factors influence the dynamical properties of power systems. In particular, parameters critical for the stability, such as lumped inertia or damping of the system, are highly dependent on the ratio of renewable energy sources in the power generation mix. As a consequence, the dynamical properties of power systems are changing on a daily basis while uncertainties in the system, i.e., the frequency and amplitude of disturbances, are significantly increased. Hence, the secondary controller tuning on a yearly basis, as done nowadays, will fail to maintain the current balancing performance in the future power systems.

The economical and physical layers of power systems are highly coupled. In addition, the time frames of both energy, and ancillary service markets, start to overlap with the time frames of physical processes. An example of the market influence to the physical behaviour of the overall power system is shown in Fig. 1, where significant frequency deviations at the program time unit (PTU) crossings are shown on two different systems. This phenomenon is widely recognized and (a) Current wind
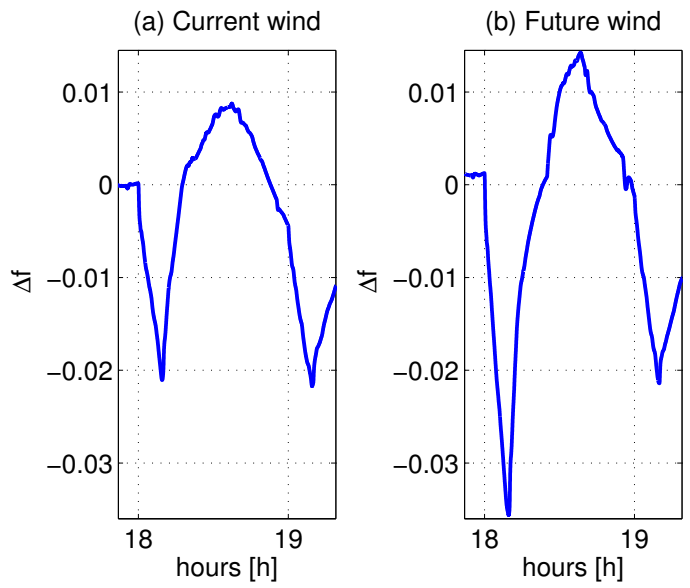

Fig. 1. Frequency deviations at the hourly crossing in the evening hours, with, on the left, the current amount of wind energy (year 2010) and, on the right, the future amount of wind energy as predicted in the year 2030 .

seen as one of the challenges in the power system operation, [1], [2], [3]. As frequency deviations at the hourly crossing occupy $70 \%$ of the primary control reserves, see [1], the system is not resilient to the disturbances that take place at the time. Although undesirable from the performance point of view, the hourly transients illustrated in Fig. 1 are useful as they give insights in the system dynamics, for instance the lumped inertia and damping. The larger the inertia, the smaller and slower the frequency drop is. The frequency deviation of the current and future Dutch system are illustrated, on the left and right side of Fig. 1, respectively. Due to higher contribution of wind farms in the generation mix, the inertia in the system in 2030 is lower, and it is manifested in the frequency response (right side) as steeper and larger deviations at the hourly crossing. Similarly, in a system with no implemented secondary control, the steady-state frequency deviation gives information about the system damping. The described changes require proper daily secondary controller tuning, for which high-quality up-to-date models are needed.

In this paper, we present a procedure for obtaining a loworder model that captures the dynamics of a control area relevant for the transmission system operator (TSO). The main contribution lies in utilization of the information on the energy market outcome, which is an autonomous signal known in advance, to identify the dynamics of a power system. This has 
not been exploited in the past. By rethinking the interconnected power systems in the system identification framework, it becomes possible to obtain a consistent model of a control area within a few hours. Next to the control area dynamics, the influence of the rest of the interconnected system is identified. The convergence of the identification algorithm is such that daily changes in dynamical properties of power systems can be captured. It is expected that a controller based on such a model is more robust to the daily changes in power system dynamics, and moreover, capable of improving the system performance. A discussion on several identification methods is provided with the intention to illustrate the challenges, difficulties and peculiarities of the given problem. Several illustrative examples show the validity of the proposed approach. In the examples a validated model of a Dutch market and power system, as described in [4], which has been continuously developed by DNV KEMA ${ }^{1}$, has been utilized.

\section{PROBLEM FORMULATION}

In this paper, we are concerned with the following problem: under which assumptions, from which signals and with which method is it possible to identify the dynamics of a control area for the purposes of the power system secondary controller? The dynamics of interest is defined by the specifications of the secondary controller which has to operate based on the realtime measurements sampled every $4 \mathrm{~s}$. As the measurements sampling time is inherently $4 \mathrm{~s}$, faster dynamics cannot be captured. Primary control is exclusively responsible for the frequency range $f \in\left[\frac{1}{2} f_{s}, \bar{f}_{P C}\right)$, but it also contributes in the lower frequency range. Faster dynamics are, in fact, less relevant for the secondary controller, [5]. Therefore, we define the frequency range of interest as the low frequency range in interval $f \in\left[0, \frac{1}{2} f_{s}\right)$, where $f_{s}=\frac{1}{T_{s}}=0.25 \mathrm{~Hz}$ is the sampling frequency, and $\frac{1}{2} f_{s}=0.125 \mathrm{~Hz}$ is the Nyquist (Shannon) frequency.

The problem can now be formulated as a closed loop networked system identification problem:

Identification of a power system control area for the purposes of secondary control

Consider a control area, which is a part of an interconnected power system. Given area control error ( $A C E)$ and secondary control requests from the secondary controller $\left(\Delta p^{S C}\right)$, find a consistent, black-box model of the dynamics of a control area for the purposes of real-time secondary controller synthesis, i.e., in frequency range of interest, $f \in\left[0, \frac{1}{2} f_{s}\right)$. It is required that convergence of estimated model is obtained within a few hours, as the goal is to capture the daily patterns in physical phenomena.

\section{POWER SYSTEM MODELLING}

\section{A. Physics of a power system control area}

A power system is a large-scale interconnected system which is administratively, and for purposes of control, divided into independent control areas. For the sake of simplicity, in

${ }^{1}$ For more information about DNV KEMA, visit http://www.dnvkema.com the remainder of this paper, we focus on the special case when the considered control area is connected to one neighbouring control area. Fig. 2 shows the linearized model of an interconnected power system divided into two control areas. The block $G_{i}(s)$ represents the lumped transfer function of generators inside the area. This block typically contains a second or third order linearized transfer function. Block $M_{i}(s)$ consists of a first order transfer function, $M_{i}(s):=\frac{1}{J_{i} s+D_{i}}$, where $D_{i}$ is a damping constant, and $J_{i}$ equivalent inertia of the control area. $T_{i j}$ is a tie-line reactance between the considered control area $\mathcal{A}_{i}$ and its directly neighboring area $\mathcal{A}_{j} \cdot \frac{1}{R_{i}}$ is a proportional frequency controller with $R_{i}$ being referred to as droop or speed regulation. The positive parameter $\beta_{i}$ denotes the composite frequency response characteristic, and has a value which is in close relation with the aggregated damping and inverse droop value of the considered control area. $\Delta q_{i}$

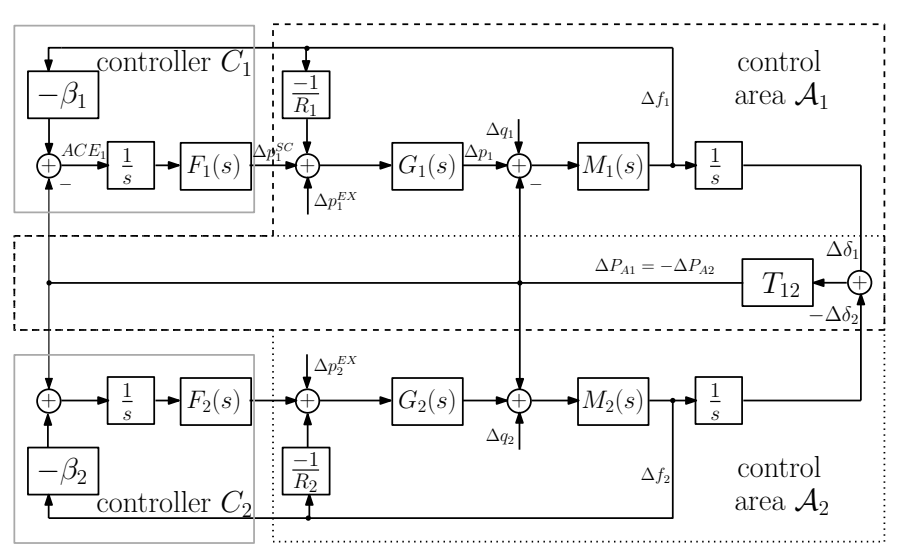

Fig. 2. Linearized block scheme of two connected control areas.

is the load disturbance, and $\Delta \delta_{i}$ is a voltage angle deviation from the nominal value of the control area $\mathcal{A}_{i}$.

Finding a model structure, model order and accurate model parameters of a power system is a difficult task. For several reasons, power system identification is recognized as a challenging problem, [6], [7], [8]. Firstly, a power system is a complex, high order, nonlinear, time-varying, closed loop system with many heterogeneous subsystems. Secondly, many random nonmeasurable disturbances excite the system and there is no easily controllable and/or measurable external excitation signal. Many disturbance signals (such as load disturbance, or wind disturbance) interfere with the "useful" signal used as the reference in the system identification. In other words, the signal to noise ratio is critical. Lastly, the model of interest is not the complete model of the interconnected power system, but only the model of the power system as the secondary controller of the considered control area experiences it; i.e., only a part of the interconnected, large-scale system. Thus, system identification is performed in an interconnected environment, of which the considered control area is only a part.

Identification of a control area gives insights not only in the dynamics inside the control area, but also in the interacting dynamics of the rest of the system, i.e., it captures the 
dynamical influence of other control areas to the considered control area. For control purposes, this is of special interest, as the system to be controlled operates as a part of a networked system. Identification enables a controller to adapt to slow changes inside the control area and the influences of other control areas, including changes in parameters in other areas and influences of other secondary controllers.

\section{B. Economical features of a power system control area}

Electrical energy is traded on wholesale energy markets or bilaterally in temporal blocks defined by the market interval, the PTU. In Europe, the market defined time interval lasts for one hour or a quarter of an hour, depending on the market design of a considered control area. Within the PTU, the generator setpoints, $\Delta p_{1}^{E X}$ are fixed, which is a consequence of the block-wise energy trade. In each market defined interval, the generator setpoints nominally change stepwise. This stepwise change causes huge frequency deviations at the PTU crossings, as previously illustrated in Fig. 1. Although numerous market parties utilize the knowledge of the setpoint from the upcoming PTU, and start changing the setpoints before the ending of a PTU, it is unavoidable that the system is additionally excited during the PTU crossings. Markets, both, wholesale and bilateral, determine the generators setpoint, and, hence, the operating point, which in turn determines the model parameters of a linearized power system.

\section{Control of a power system control area}

Due to the inability of efficiently and economically storing large quantities of energy, the energy supply necessarily has to meet the demand in real-time. The secondary controller, $C_{1}$ in Fig. 2, of the control area $\mathcal{A}_{1}$, is in charge for keeping the global balance between production and consumption within the considered control area, and scheduled tieline flows at the agreed level. The control goal is to reduce the influence of disturbances, which are usually a consequence of inaccurate load or wind predictions. The controller reacts to the $A C E$ signal of its own control area. The $A C E$ is a linear combination of frequency deviation and power flow deviation defined as $\operatorname{ACE}(s):=\beta_{1} \Delta f_{1}(s)+\Delta P_{A 1}(s)$. The controller is generally of a proportional plus integral (PI) structure.

$$
\Delta p_{1}^{S C}(s):=-\left(K_{p}+\frac{K_{i}}{s}\right) A C E(s),
$$

where $K_{p}$ is typically chosen to be zero or very small.

In addition to the PI controller, the secondary controller contains an anti aliasing and a low pass filter. The anti aliasing filter is necessary to avoid the aliasing caused by the low sampling time of 4 seconds. The output of the low pass filter, usually implemented in discrete time domain with a pole around 0.9, is known in literature as the processed $A C E$ signal, $P A C E$. The frequency and time ranges of interest are shown in Fig. 3. Here, the word frequency refers to the frequency domain in which the data is processed, and not to the power system frequency. The most right frequency shown in Fig. 3 is the Nyquist frequency, defined as the frequency which is twice as small as the sampling frequency. Above

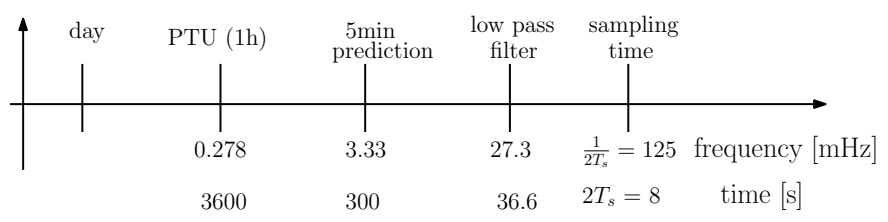

Fig. 3. Frequency and time ranges of interest.

this frequency, due to the chosen sampling time, it is not possible to make conclusions about the system dynamics. In the considered application, the sampling time is defined by the controller sampling time, which is $4 \mathrm{~s}$, and corresponds to the Nyquist frequency of $125 \mathrm{mHz}$.

The following frequency of interest is the frequency associated with the low-pass filter of the controller, which is generally of the form:

$$
P A C E(k):=0.1 A C E(k)+0.9 A C E(k-1) .
$$

This filter has a pole at $z_{L P}=0.9$ in the discrete domain. Translated to the continuous time domain, the pole is at $s_{L P}=$ $e^{-\frac{1}{2 \pi} T_{s} z_{p}}$, which corresponds to frequency of $27.3 \mathrm{mHz}$. As this filter has smoothened all the higher frequencies, the information for the frequency range $f \in\left[0, e^{-\frac{1}{2 \pi} T_{s} z_{p}}\right)$ can be obtained with accuracy.

Next, we consider the situation in which the market agents, or, as called widely in Europe, the balance responsible parties (BRPs) act based on the knowledge that they have on the market outcome. This knowledge is available from the blockwise, PTU based trade. It is reasonable to assume that the generators do not change the setpoint block-wise, as it is physically challenging and undesirable, but that they use the a priori knowledge on the future situations to adjust the generator setpoint before the agreed hourly change. Assuming that this happens roughly 5 minutes before the PTU change, we obtain the next frequency of interest: $f_{\text {pred }}=3.33 \mathrm{mHz}$. It is expected to see the effect of predictive behaviour at around this frequency in the frequency domain.

The last frequency of interest in the frequency related to the PTU duration, i.e. $f_{P T U}=0.278 \mathrm{mHz}$. At this frequency, it is possible to see periodicity of the market signal spectrum.

\section{Signals in the power systems}

The following list defines and classifies the relevant signals in a control area, as presented in Fig. 2:

TABLE I

RELEVANT SIGNALS AT THE CONTROLLER LEVEL.

\begin{tabular}{|c|l|c|}
\hline signal & description & sampling time, $T_{s}$ \\
\hline \hline$\Delta f_{1}$ & $\begin{array}{l}\text { frequency deviation from the nominal } \\
\text { value }\end{array}$ & $4 \mathrm{~s}$ \\
\hline$\Delta P_{A 1}$ & $\begin{array}{l}\text { tie-line power flow deviation from the } \\
\text { nominal value }\end{array}$ & $4 \mathrm{~s}$ \\
\hline$\Delta p_{1}^{S C}$ & secondary control setpoint & $4 \mathrm{~s}$ \\
\hline$\Delta p_{1}^{E X}$ & market defined generator setpoint & $900 \mathrm{~s}$ (or 3600s) \\
\hline
\end{tabular}

An indication or a measurement of signals $\Delta f_{1}, \Delta P_{A 1}$, $\Delta p_{1}^{S C}$ and $\Delta p_{1}^{E X}$ is available to the controller in real-time. 
Note that the market defined generator setpoint is a stepwise signal which changes the value every 900s (or 3600s). Hence, for this signal, Tab. I gives the time between the two steps instead of the sampling time.

\section{Selection of System identification MEthod}

In this section, we present a control area as defined above in the system identification framework, together with a survey of several closed loop system identification methods that are applicable for identification of power system control area dynamics.

One of the system identification goals is to obtain a consistent estimate. Consistency guarantees that, whenever the model set contains the real system (the data generating system), under additional assumptions on excitation of the reference signals and a number of samples, the identified model corresponds to the real system, [9].

There are two main reasons that contribute to the complexity of the task of obtaining the high-quality power system control area models: $(i)$ power systems operate in a closed loop with no possibilities of introducing an external excitation signal, which might result in insufficiently informative data, and (ii) a control area is a part of an interconnected environment.

\section{A. System identification framework representation of a control area}

In the system identification framework, signals are commonly grouped into input signals, output signals, disturbances, and reference signals. In case of closed-loop identification, such as power system identification, the existence of a persistently exciting reference signal is crucial for obtaining consistent models. The signals in a control area are grouped as follows:

$$
\begin{aligned}
\Delta f_{1}, \Delta P_{A 1} & \mapsto \mathcal{Y} \\
\Delta p_{1}^{S C} & \mapsto \mathcal{U} \\
\Delta q_{1} & \mapsto \mathcal{E} \\
\Delta p_{1}^{E X} & \mapsto \mathcal{R}
\end{aligned}
$$

where $\mathcal{Y}$ is a set of output, $\mathcal{U}$ set of input, $\mathcal{R}$ set of reference signals, and $\mathcal{E}$ set of disturbances. The set of reference signals, $\mathcal{R}$, contains the external signals which have to be uncorrelated with the set of disturbance signals, $\mathcal{E}$. Non existence of correlation among the two is a crucial assumption in obtaining consistent estimates under the closedloop identification. Market signal $\Delta p_{1}^{E X}$ is perhaps the only autonomous signal uncorrelated with the disturbances in power systems. The choice $\Delta p_{1}^{E X} \mapsto \mathcal{R}$, with $\Delta p_{1}^{E X}$ an autonomous, measurable signal, is a novelty in the field of power system identification and it makes possible applying the established system identification methods to the power system control area identification problem. Another important assumption on the reference signals is the persistency of excitation. Note that, in the considered case, there is no freedom in shaping the reference signals, meaning that it is not possible to influence the persistency of excitation. This is one of the biggest limitations in applying system identification methods to the power systems.

Exploiting the economically generated setpoints, i.e., a known, measurable signal $\Delta p_{1}^{E X}$, enables application of the well established system identification methods for obtaining a consistent model of a control area. This is in contrast with the current practice, where models are based on operator's experience and previous knowledge of the system. Note, however, that implicitly, assumption on the PTU duration and decoupled operation of day-ahead markets and real-time processes has been made. There are market designs with shorter PTUs, e.g., five minutes in Australia. The proposed method can deal with this situation as well. In fact, shorter PTU lengths imply faster changes in the reference signal. This is beneficial, as the changes directly influence the persistency of excitation and hence the identification convergence. Nevertheless, the proposed approach is not suitable for the market designs with incorporated locational marginal pricing or real time markets, such as PJM energy market. In such markets, the time gap between the day-ahead and real-time operations is significantly smaller and, depending on the system dynamics, the market signal, $\Delta p_{1}^{E X}$, becomes dependant on the system state, and as such cannot be considered as autonomous any more. In this case, the assumption on independence of the market signal is violated.

The configuration of a control area operating in closed loop, which is convenient for system identification analysis, can now be shown as presented in Fig. 4. Here, $u$ is input, $y_{1}, y_{2}$ and

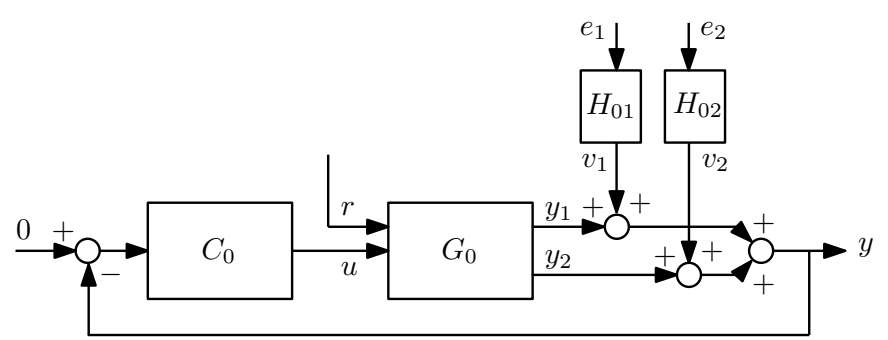

Fig. 4. Representation of a control area in the system identification framework.

$y$ outputs, $e_{1}$ and $e_{2}$ noise and $r$ external excitation signal. Mapping Fig. 2 to Fig. 4, we define:

$$
\begin{array}{r}
r:=\Delta p_{1}^{E X}, \quad u:=\Delta p_{1}^{S C}, \quad y:=A C E_{1}, \\
y_{1}:=\beta_{1} \Delta f_{1}, \quad y_{2}:=\Delta P_{A 1} .
\end{array}
$$

We define the following transfer functions of interest: $G_{1}(s):=\frac{A C E_{1}(s)}{\Delta p_{1}^{E X}(s)}, G_{2}(s):=\frac{\Delta p_{1}^{S C}(s)}{\Delta p_{1}^{E X}(s)}$, and $G_{0}(s):=$ $\frac{A C E_{1}(s)}{\Delta p_{1}^{S C}(s)}$. From the above scheme, it is straightforward to obtain the expression for obtaining $G_{0}(s)$ from $G_{1}(s)$ and $G_{2}(s)$ as

$$
G_{0}(s):=\frac{G_{1}(s)}{1+G_{2}(s)} .
$$

In the remainder of this section, a brief overview of the utilized closed loop system identification method is given. The 
inventory presented below is by no means exhaustive. For a complete survey of system identification methods for closed loop systems, see e.g. [10].

\section{B. Prediction error identification methods}

The most suitable method for the given problem is a prediction error method (PEM), described in [9] and [11]. One of the prerequisites to applying the prediction error methods (PEM) is the persistency of excitation of the reference signal. The notion of persistency of excitation is defined for finite power signals, and as such not suitable for signals such as step signals of length much longer than the time necessary for the system dynamics to settle. Slightly abusing the assumption on persistency of excitation, it is possible to apply PEM to the considered problem. After sufficient number of changes in the step-wise reference signal, a consistent estimate is obtained. Other methods, such as frequency response function estimation, [9], or transient response identification, [9], also exist. Frequency response function estimation is suitable for periodic reference signals, which is clearly not the case in the considered problem. Transient response identification is suitable if the system reaches the steady-state before the next change in the reference signal. As in current power systems this was not the case, we do not consider this method in this paper.

The result of a PEM identification method is a transfer function with a limited number of coefficients, which captures the characteristic dynamical phenomena of the system. It is suitable for control design and simulation. The PEM is based on the least squares algorithm, which finds the optimal time domain curve fitting for the given transfer function model, i.e., for the prespecified number of parameters.

To run a PEM method, a model structure, delay and model orders have to be defined a priori. The most general model structure is the Box-Jenkins (BJ) model, where output $y(k)$ is modeled as a linear combination of response to input $u(k)$ and to the disturbance signals $e(k)$ :

$$
y(k)=\frac{B(q, \theta)}{F(q, \theta)} u(k)+\frac{C(q, \theta)}{D(q, \theta)} e(k),
$$

where $\theta$ is the vector of the model parameters, and $q$ is the shift operator. The latter equation can take matrix form so that the dynamics of multiple input multiple output processes can also be estimated. Output error (OE) model is of the same structure as BJ model, but without explicit noise modeling, i.e.:

$$
y(k)=\frac{B(q, \theta)}{F(q, \theta)} u(k)+e(k) .
$$

The goal of the PEM method is to minimize an identification criterion $J(\theta)$, commonly defined as normalized sum of prediction errors $\epsilon(k, \theta):=y(k)-\hat{y}(k, \theta)$ :

$$
\min _{\theta} J(\theta)=\min _{\theta} \frac{1}{N} \sum_{n=1}^{N} \epsilon(k, \theta) .
$$

Both discussed model structures are such that the identification algorithm results in a nonlinear optimization. This method is convenient for the recursive implementation, so online identification based on PEM algorithm is possible. The theory on the model validation is well developed, [9], with methods such as residual analysis being implemented as part of MATLAB system identification toolbox.

\section{Procedure}

In this paper, we applied the prediction error method with OE and BJ model structures to obtain an estimated model of a power system control area. The following procedure has been employed:

1) From the data, the transfer functions $G_{1}(s)$ and $G_{2}(s)$ are obtained using one of the above described identification methods.

2) From the transfer functions obtained in the first step, the desired transfer $G_{0}(s)$ is obtained using equation (1).

Step 2) of the procedure, where the division of two obtained models is performed, can cause the final model to be unstable, even if both models from step 1) are stable. In case of noisy data, it is important to have long time series to improve the signal to noise (SNR) ratio. However, if the system parameters change over time, the data sequences should be limited to the period in which the system parameters were constant. If the algorithm is implemented recursively, it is possible to assign different weights to the data by choosing the so-called forgetting factor to be less than 1 . In such way, the newer data can be assigned higher weight than the data measured longer ago, while still keeping long time series to improve the signal to noise ratio.

\section{EXAMPLES AND DISCUSSION}

In this section, we present the obtained experimental results on two cases. In Case 1, the power system model given in [12] and presented in the Fig. 2 has been considered. There are two examples illustrated. In the first example of Case 1, the validity of the chosen approach has been illustrated by showing that the PEM method gives good estimation results, despite slight violation on persistency of excitation assumption. In the second example of the Case 1, we show how quickly the identification method catches the model changes after decrease in one of the parameters, namely inertia $J_{1}$. In Case 2 , identification process is based on data obtained from the validated model of the Dutch power system, [4], which has been continuously developed by DNV KEMA over more than a decennium. The model incorporates not only the physical, but also the economical layer and as such is unique and much more complex than the model presented in textbooks.

\section{A. Case 1}

To test the algorithm, the following values have been assigned to the parameters of the system shown in Fig. 2: $J_{1}=J_{2}=10, \quad D_{1}=D_{2}=1, \quad R_{1}=R_{2}=0.05, \quad G_{1}=$ $G_{2}=\frac{2.1 s+1}{0.42 s^{3}+3.56 s^{2}+7.5 s+1}, \quad \beta_{1}=\beta_{2}=21, \quad T_{12}=7$. The 
sampling time has been chosen to be the same as in practice, $T_{s}=4 \mathrm{~s}$. Hence, the real transfer function of the process is:

$$
\begin{aligned}
G_{o}= & \frac{0.80409(z-0.8555)(z-0.5595)(z-0.5502)}{z\left(z^{2}+0.09602 z+0.02169\right)} \\
& \frac{(z-0.2447)(z+0.1102)\left(z^{2}-1.139 z+0.3245\right)}{(z-0.8531)(z-0.6544)(z-0.5647)^{5}} \\
& \frac{\left(z^{2}-1.125 z+0.3164\right)\left(z^{2}-0.399 z+0.07714\right)}{\left(z^{2}-0.5984 z+0.1221\right)} .
\end{aligned}
$$

The goal is to find the model relevant for the secondary controller, i.e., the transfer function $G_{0}$, using 4 second measurements of $\Delta p_{1}^{S C}, A C E_{1}$ and $\Delta p_{1}^{E X}$, for an arbitrary chosen input sequence $\Delta p_{1}^{E X}$.

1) Example 1.: Let all the parameters be as given above. A load disturbance, in form of disturbance signal $\Delta q$, has been simulated as white noise. The data is collected over four PTUs. The sampled measurements are shown in Fig. 5, where

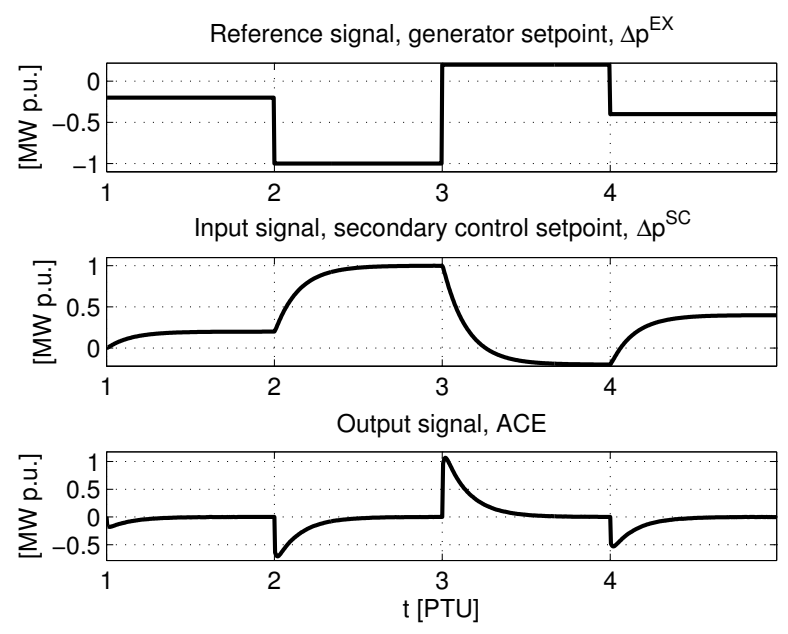

Fig. 5. Case 1, representation of relevant signals in the time domain.

the time samples of an arbitrary chosen market reference (Eprograms), $\Delta p_{1}^{E X}$ (subplot 1), the secondary controller output, $\Delta p_{1}^{S C}$ (subplot 2), and the area control error, $A C E_{1}$ (subplot 3 ), are illustrated. The effect of the white noise is not obvious in these plots due to its relatively small variance, but it is visible in the frequency domain, in Fig. 6, in form of spikes. The influence of the white noise is especially visible on higher frequencies (closer to the Nyquist frequency), as with increase in frequency, the SNR in this particular example decreases.

Fig. 6 shows the estimated model compared to the real transfer function and the frequency representation of the $G_{0}(s)$ transfer. Note that the identification method resulted in a very good model representation of the real transfer function (almost perfect overlap in the Bode diagram). This is expected, as the SNR in the considered example is favourable.

2) Example 2.: Next, consider a case in which the power system dynamics changes during the experiment. During the PTU 5, inertia in the considered control area, $J_{1}$, drops to $25 \%$ of its nominal value. The identification algorithm has
Comparison of measured data, estimated model and real system

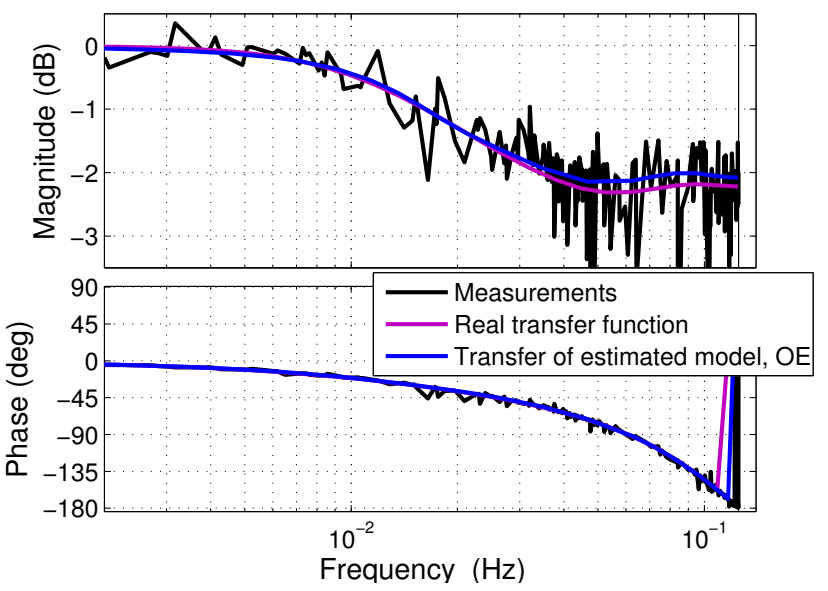

Fig. 6. Case 1, comparison of measurement represented in frequency domain, real transfer function and obtained estimate.

been tuned so that it does not take into account measurements older than 5 hours. This can be achieved by assigning the forgetting factor in the recursive identification algorithm, or simply by discarding the data. The latter is (almost) never advised to do in system identification.

The results are shown in Fig. 7. The convergence has been obtained within 3 PTUs, Fig. 7(a). After the value of lumped inertia in the system changed, during the 5th PTU, there is an increase in model variance and a clear discrepancy between the real and estimated model, Fig. 7(b). With more time and more measurements, the estimated model becomes closer to the real model, Fig. 7(c), and after some time it obtains the real model parameters, Fig. 7(d). In this particular example, depending on the SNR, the convergence has been obtained within 3 to 4 PTUs, which is sufficiently fast for the controller to adapt to the daily changes in power system dynamics.

\section{B. Case 2}

In case study 2, we tested the proposed approach on the data obtained from the validated model of the Dutch power system coupled with the market layer. The day-ahead market results are fed into the power system model which consists of 46 generator units, 23 wind plants and load divided over 7 BRPs. The model is based on the physical properties of the Dutch power system in the year 2010. This model, in contrast to the model used in Example 1, is a highly complex, nonlinear model. The nonlinearities come from the generator ramp rate constraints and saturations. Additionally, depending on the PTU, the bid ladder for the secondary control changes, so for the same $A C E$ value, in different PTUs, the TSO might request reserves from different market participants and hence activate different dynamics. The real low order transfer function from $\Delta p_{1}^{S C}$ to $A C E_{1}$ is not accessible. The obtained model has to be validated by residual analysis. The residual analysis can justify the chosen model order and structure. In these experiments, 4 second measurements of $\Delta p_{1}^{S C}, A C E_{1}$ and $\Delta p_{1}^{E X}$ have 


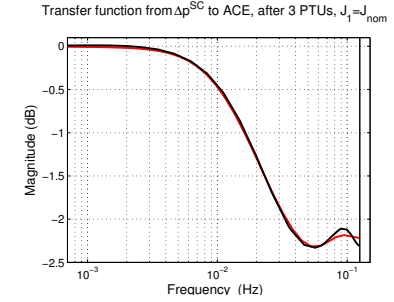

(a) Obtained estimate after 3 PTUs, with $J_{1}=J_{\text {nom }}$.

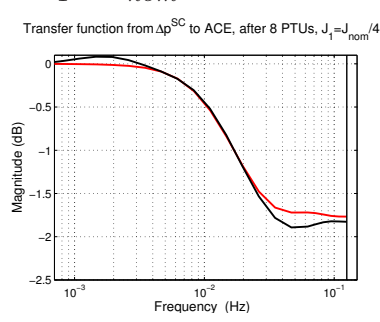

(c) Obtained estimate after 8 PTUs, with $J_{1}=0.25 J_{\text {nom }}$.



(b) Obtained estimate after 6 PTUs, with $J_{1}=0.25 J_{\text {nom }}$

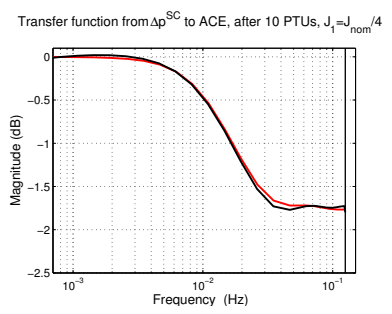

(d) Obtained estimate after 10 PTUs, with $J_{1}=0.25 J_{\text {nom }}$.

Fig. 7. Case 1, Example 2, illustration of the algorithm convergence in case of changes in system dynamics. Red line represents the real model, and black one the estimated.

been collected over the period of 24 hours. The identification is performed a posteriori, and not recursively. Identification algorithm has been conducted on data of various lengths, e.g., first after first 3 hours, then after first 5 hours and so on. The purpose is to simulate the convergence of the algorithm as the time progresses. However, for validation purposes, we always utilized the full data sequence (full $24 \mathrm{~h}$ measurements).

Fig. 8 shows the measured data in dependance on time. Obviously, the collected data is subject to noise. This is
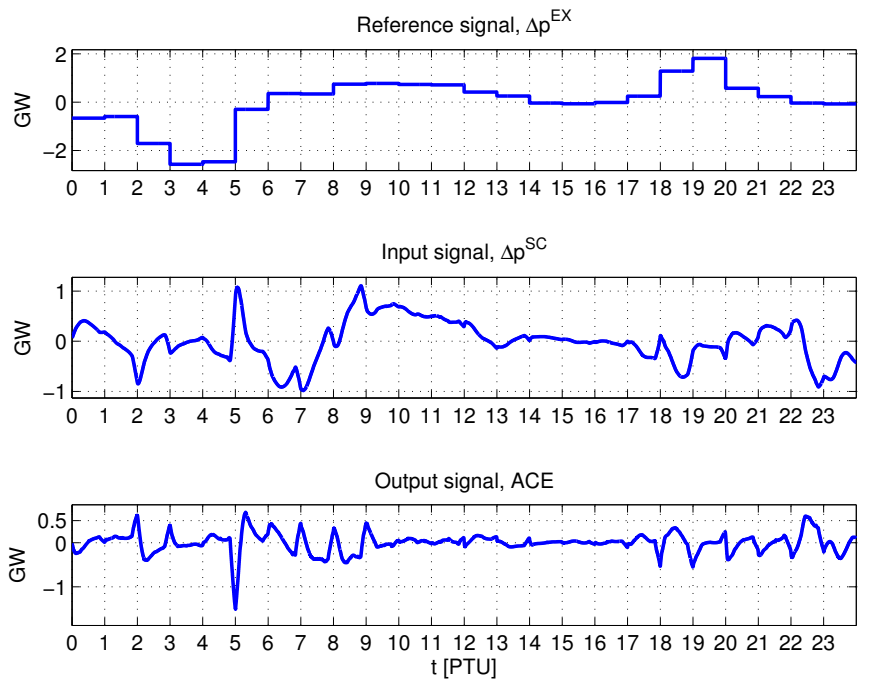

Fig. 8. Case 2, representation of relevant signals in the time domain

illustrated in detail in Fig. 9, where discrete Fourier transformations of the reference signal, $\Delta p_{1}^{E X}$, and noise signals (wind and load disturbances) is presented. The Fourier transformation is chosen, as it provides good insights into signal and noise spectra and, hence, signal to noise ratio. Fig. 9 shows that low frequencies experience noise spectrum which is comparable in size with the spectrum of the reference signal, and with the increase in frequency values, for the middle frequency range, this ratio gets better in favor of signal, see zoomed sections in Fig. 9. On frequencies close to the Nyquist frequency, spectra of noise and reference signal get comparable again. Hence, it is expected that the estimated transfer function contains certain steady state bias compared to the real model, as well as inferior modelling around the sampling frequency.

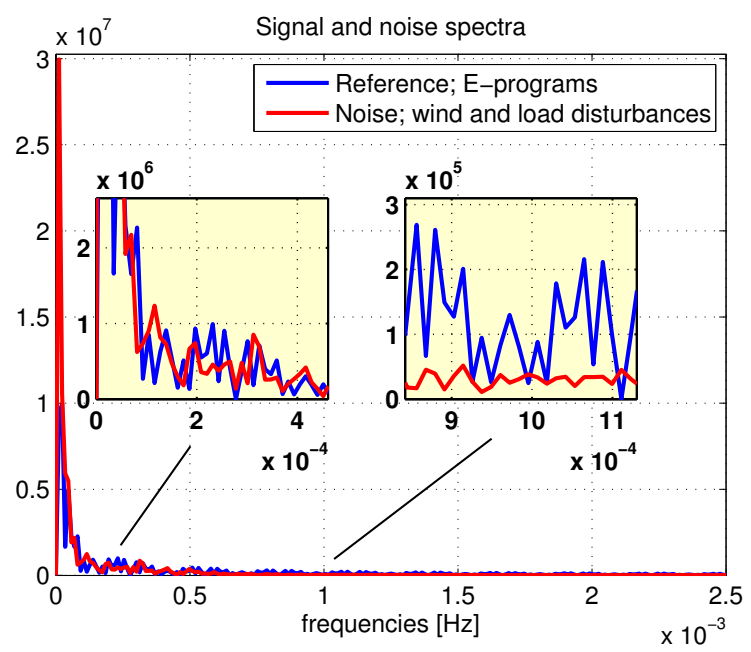

Fig. 9. Case 2, signal and noise spectra.

We conducted the PEM identification for the OE model structure, equation (3), with $B(q, \theta)$ of 7 th order, and $F(q, \theta)$ of 8 th order, i.e. $n_{p}=8, n_{z}=7$. To illustrate the convergence of the identification method, we conducted 5 experiments in total: on the data collected over first 3, 7, 12, 17 and 24 hours. Hence, there are 5 different models obtained. To illustrate the quality of the obtained models, we compared the outputs of these 5 models with the measured output. Although the data used for identification varied, for model validation all the measurements ( $24 \mathrm{~h}$ data) has been used. The comparison of the measurements and model estimated OE models are presented in Fig. 10. To validate the obtained models, it is necessary to perform the residual analysis, which showed that satisfactory model and model orders have been chosen. The plots are omitted for brevity.

Next, we conducted the PEM identification for the BJ model structure, equation (2), to explicitly model the noise dynamics in the system. For consistent modeling, it is necessary that both the process, and the noise model are consistent. The chosen orders, for which the residual analysis validates the results, are $B(q, \theta)$ of 7 th order, $F(q, \theta)$ of 8 th order, $C(q, \theta)$ of 13 th order, $D(q, \theta)$ of 14 th order, i.e. $n_{p}=8, n_{z}=$ $7, n_{c}=13, n_{d}=14$. Fig. 11 shows the convergence of the identification algorithm. The identification procedure based on data obtained after 1,2 or 3 hours yields models whose output deviates significantly from the measured output, and 


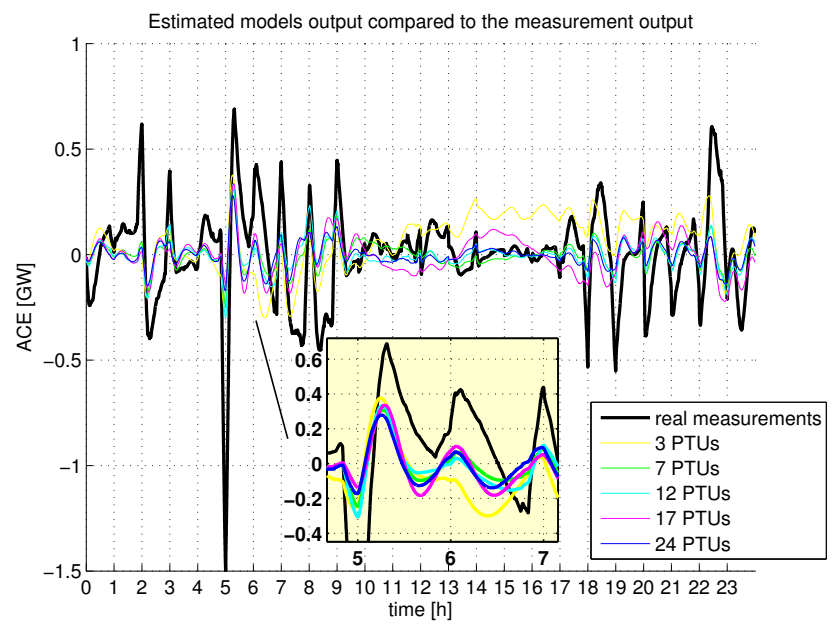

Fig. 10. Case 2, comparison of the measurements and model estimated with OE model after $3,7,12,17$ and 24 hours. Chosen model order is $n_{p}=$ $8, n_{z}=7$

are not illustrated in the figure for clarity reasons. This is explained by the low signal to noise ratio, i.e. insufficiently long data sequence. In the considered example, after the 4 PTUs, the measurements are sufficiently informative for the identification procedure to return a model that can well mimic the real system. With more data, the estimated model gets better and better. In conclusion, from the data obtained from

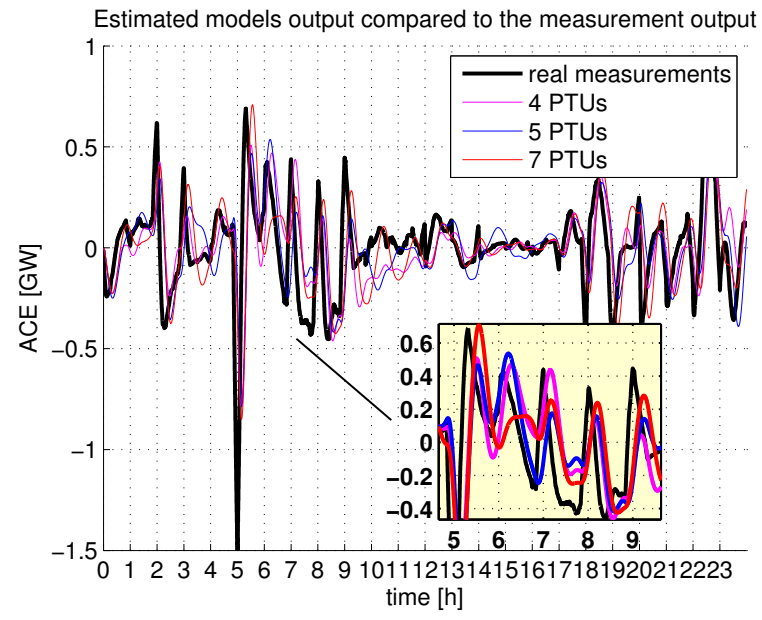

Fig. 11. Case 2, convergence of the model estimate with BJ model after 4,5 , and 7 hours. The identification procedure based on data obtained after 1,2 or 3 hours yields models with large variance. Chosen model order is $n_{b}=7, n_{f}=8, n_{c}=13, n_{d}=14$.

the validated Dutch power system model, it was possible to obtain an estimated model utilizing the prediction error method with the PEM. Depending on the chosen structure, different low order models are obtained. If it is important to model the noise dynamics, one should go for a BJ model, otherwise OE structure is sufficient.

\section{CONCLUSIONS}

In this paper, the power system control area is presented in the context of the system identification. We argued that with the current changes in power systems, in the future the existence of high-quality up-to-date models will become increasingly important for performance and reliability of daily operation. Online recursive system identification is necessary to obtain such models. However, due to the limited amount of data, and many excitation signals in power systems with relatively low signal to noise ratio in the frequency range of interest, and operation in an interconnected environment under the closed-loop conditions, it is challenging to obtain a model that satisfies the requirements. We have shown that using the market signal, which is in the power system context one of the very few, if not the only, autonomous measurable signals uncorrelated with the noise, it is possible to obtain a low order approximation model of a control area. Such a model can serve as a basis for the secondary controller design. The proposed method is illustrated on two examples. One of the examples is based on the data from the validated model of a Dutch power system.

\section{REFERENCES}

[1] Tractebel, "Study of the interactions and dependencies of balancing markets, intraday trade and automatically activated reserves," Tractebel, Tech. Rep., 2009. [Online]. Available: http://ec.europa.eu/energy/gas_electricit y/studies/doc/electricity/2009_balancing_ markets.pdf

[2] Z. W. Li, O. Samuelsson, and R. Garcia-Valle, "Frequency deviations and generation scheduling in the nordic system," in 2011 IEEE Trondheim PowerTech. IEEE, 2011.

[3] ENTSO-E, "Ucte operation handbook," Tech. Rep., 2004, last accesed: January $2012 . \quad$ [Online]. Available: https://www.entsoe.eu/resources/publications/systemoperations/operation-handbook/

[4] G. Dekker, J. Frunt, W. de Boer, and M. Duvoort, "Case studies and results of the e-price approach in power systems," in Proceedings of the 10th International Conference on the European Energy Market, EEM13, Stockholm, Sweden, 2013.

[5] N. Jaleeli, L. VanSlyck, D. Ewart, L. Fink, and a.G. Hoffmann, "Understanding automatic generation control," IEEE Transactions on Power Systems, vol. 7, no. 3, pp. 1106-1122, 1992.

[6] I. Vajk, M. Vajta, L. Keviczky, R. Haber, J. Hetthéssy, and K. Kovács, "Adaptive Load-frequency Control of the Hungarian Power System," Automatica, vol. 21, no. 2, pp. 129-137, 1985.

[7] J. W. Pierre, D. Trudnowski, M. Donnelly, N. Zhou, F. K. Tuffner, and L. Dosiek, "Overview of system identification for power systems from measured responses," Proc. 16th IFAC Symposium on System Identification, Brussels, Belgium, pp. 989-1000, 2012.

[8] A. Rubaai and V. Udo, "An adaptive control scheme for load-frequency control of multiarea power systems Part I. Identification and functional design," Electric Power Systems Research, vol. 24, no. 3, pp. 183-188, 1992.

[9] L. Ljung, System Identification: Theory for the User. Pearson Education, 1998.

[10] P. M. J. Van den Hof, "Closed-loop issues in system identification," Annual reviews in control, vol. 22, pp. 173-186, 1998.

[11] T. Söderström and P. Stoica, System Identification. Prentice and Hall, 1988.

[12] P. Kundur, Power System Stability and Control. McGraw-Hill Professional, 1994. 